\title{
Ottawa Knee Rule: Investigating Use and Application in a Tertiary Teaching Hospital
}

\author{
Abubakr Mohamed ${ }^{1}$, Elkhidir Babikir ${ }^{1}$, Mohamed Kamal Elbashir Mustafa ${ }^{2}$ \\ 1. Emergency Medicine, University Hospital Galway, Galway, IRL 2. Vascular Surgery, University Hospital Galway, \\ Galway, IRL
}

Corresponding author: Abubakr Mohamed, abubakr.elimam91@gmail.com

\section{Abstract \\ Background}

Knee injuries are encountered commonly in the emergency departments (EDs) in Ireland. Validated clinical decision rules such as Ottawa knee rule (OKR) can be used in acute knee injury settings to reduce the number of unnecessary radiography. Clinical judgment can be used to distinguish between suspected fractures and non-fractures in many cases; however, radiography is still routinely requested.

\section{Objectives}

We evaluated the OKRs in a high-volume tertiary teaching hospital in Ireland to determine whether the rule could be safely used to decide whether patients with acute blunt knee trauma should undergo radiography.

\section{Methods}

This was an observational study conducted in the ED over a three-month period in a tertiary referral hospital. A total of 110 patients with acute knee injuries were examined using OKR. Inclusion criteria included patients with acute knee injuries due to blunt trauma or twisting injury and patients with lacerations or contusions. Open fractures and fractures due to penetrating injury were excluded from the study.

\section{Results}

Fractures were seen in 12 (13.2\%) of the 110 patents who met the inclusion criteria. The OKR predicted all 12 fractures. Sensitivity was $100 \%$, and specificity was $39 \%$.

\section{Conclusions}

The OKR is highly sensitive for fracture in this setting and can be safely used to decide whether patients with acute blunt knee trauma should undergo radiography.

Received 06/04/2020

Review began 06/18/2020

Review ended 06/21/2020

Published 06/24/2020

(c) Copyright 2020

Mohamed et al. This is an open access article distributed under the terms of the Creative Commons Attribution License CC-BY 4.0., which permits unrestricted use, distribution, and reproduction in any medium, provided the original author and source are credited.
Categories: Emergency Medicine, Radiology, Orthopedics

Keywords: ottawa knee rule, knee injuries

\section{Introduction}

The Ottawa knee rules (OKRs) were designed to divide acute knee trauma patients into two groups: patients who are likely to have a clinically significant fracture and need radiography to rule out an injury and patients who have virtually no chance of having an important radiographically detectable bony injury [1-3]. The OKR consists of five components asking whether the patient is 55 years of age or older, has an isolated tenderness of the patella (no bone tenderness of the knee other than at the patella), has tenderness of the head of the fibula, is unable to flex the knee to 90 degrees, and is unable to bear weight both immediately and at the emergency department (ED) for four steps [4]. Patients with at least one positive answer are considered to have positive results for knee fracture and are advised to have radiography. After the development of the rule by Stiell et al. in 1995, the decision aid was validated in several clinical settings $[1,2]$. In situations where virtually every patient entering an ED with an acute knee injury undergoes radiography, even modest values for specificity may substantially reduce the number of unnecessary radiographs obtained. Therefore, the threshold for the OKR is standardized at high sensitivity and sacrifices specificity to some extent. A systematic review of 4,249 patients in six studies found that fractures can narrowly be excluded in a negative OKR $[5,6]$.

\section{Materials And Methods}

This was an observational study conducted in the ED of a tertiary hospital in Ireland over a three-month period. We reviewed all radiology requests and reports of knee radiographies in the ED prospectively over a three-month period (October to December 2019). 


\section{Cureus}

Patients were reviewed and their clinical records analyzed to establish the indication for ordering plain radiography. A total of 110 patients were identified. Patient records were reviewed to assess whether any of the Ottawa knee criteria were fulfilled when plain radiography was being ordered. The data collected was analyzed for diagnostic accuracy (sensitivity, specificity, positive predictive value, and negative predictive value) of the OKR.

\section{Results}

The study included 110 patients between 2 and 88 years of age (mean: 41.5 years). The gender distribution was equal, with a female-to-male ratio of 1:1 (Table 1). The majority of presentation was in the age group of 10 to 19 years (19.09\%) followed by the age group of 60 to 69 years (17.27\%). Undiagnosed knee injury was the most common diagnosis $(44.55 \%$; $n=49)$ followed by knee fractures $(10.9 \%)$. Knee effusion accounted for $10 \%$ of the injuries (Figure 1).

\begin{tabular}{|l|l|}
\hline Characteristics & Values \\
\hline Age (years) & $43(41.5)$ \\
Mean (median) & 47 \\
Interquartile range & \\
Gender (\%) & $50 \%(\mathrm{n}=55)$ \\
Male & $50 \%(\mathrm{n}=55)$ \\
Female & \\
Mechanism of injury (\%) & $75 \%$ \\
Fall & $8.33 \%$ \\
Twisting & $9.72 \%$ \\
Direct blow & \\
\hline
\end{tabular}

TABLE 1: Acute knee trauma patient demographics and injury description.

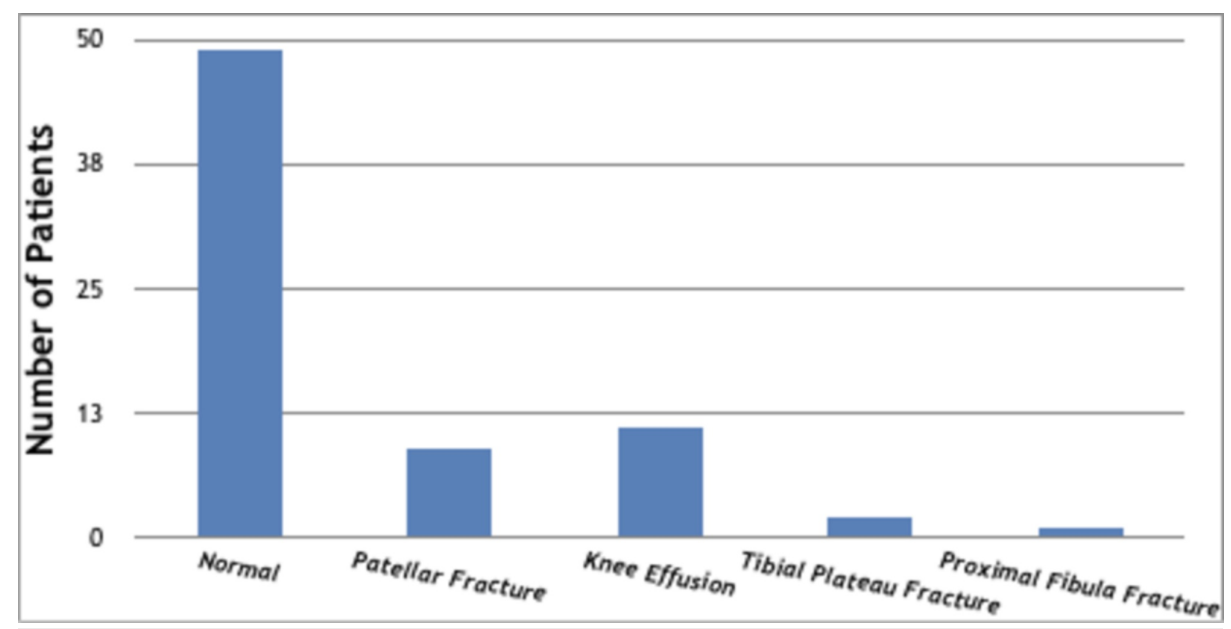

FIGURE 1: Acute knee trauma diagnosis.

Each patient's radiography request was assessed to determine whether the Ottawa criteria were fulfilled. Of 110 of request forms, 72 (65\%) were justified with enough written information to warrant an X-ray according to the OKR. The most common reason for fulfilling the Ottawa criteria was the patient age (59.72\%) followed by patellar tenderness (31.94\%), inability to bear weight (5.56\%), and fibular head tenderness $(2.78 \%)$ (Figure 2). Limited knee flexion to 90 degree was not present in all knee radiography requests. Noncompliant knee radiography had insufficient information in some cases to see whether radiography was appropriate or not, and patients who were not referred for radiography were excluded and they may have been compliant. 


\section{Cureus}

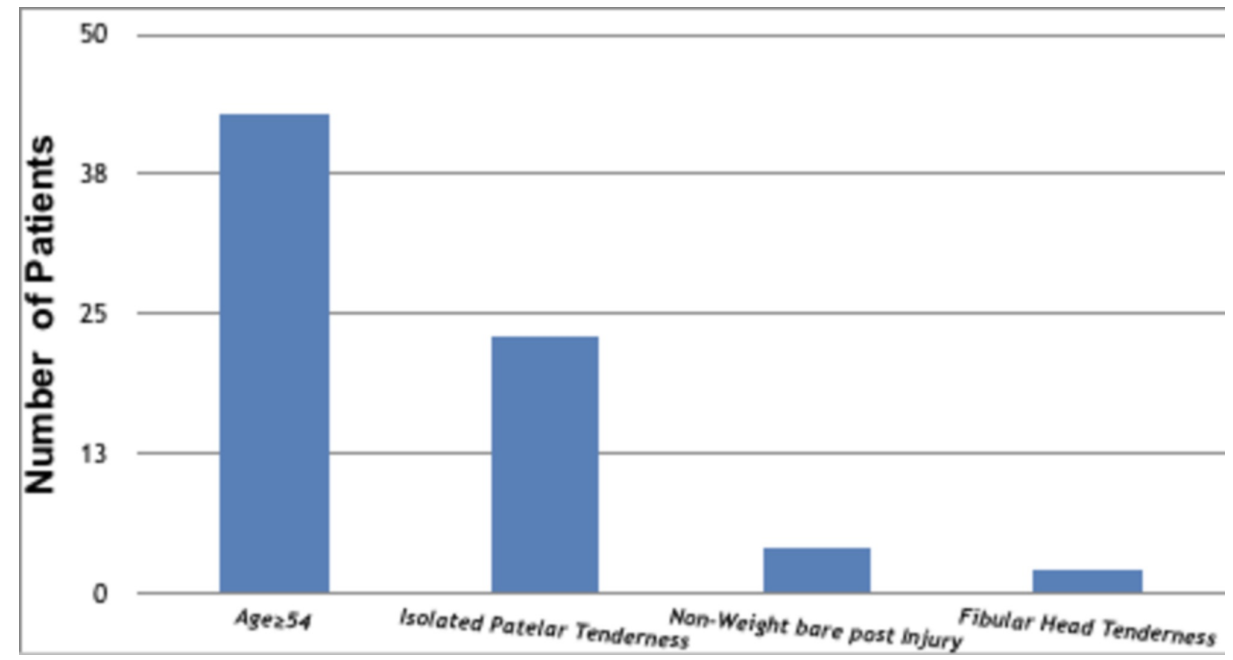

FIGURE 2: Ottawa knee rule fulfillment.

Of the patients who met the Ottawa knee criteria, $75 \%$ had a fall as their mechanism of injury followed by blunt trauma (9.72\%), twisting (8.33\%), and unknown mechanism (6.94\%) (Table 1).

Twelve patients from the study group were diagnosed with a fracture. All 12 fulfilled the OKR. Using the Ottawa rules for knee radiography, 38 radiographs (34.55\%) could have been avoided without missing a fracture. The various parameters of diagnostic accuracy for the rules are shown in Table 2. However, OKR was found to have a relatively low positive predictive value of $61 \%$ and a negative predictive value of $100 \%$.

\begin{tabular}{|l|l|}
\hline Parameters & Values (\%) \\
\hline Sensitivity & $100 \%$ \\
Specificity & $39 \%$ \\
Positive predictive value & $61 \%$ \\
Negative predictive & $100 \%$ \\
\hline
\end{tabular}

TABLE 2: Diagnostic accuracy of the Ottawa knee rules.

\section{Discussion}

Two of the most important attributes of good clinical prediction rules for fracture are high sensitivity and external validity $[2,3]$. Clinicians are uneasy using prediction rules that are not perfectly sensitive, that is, do not detect all cases partly because of medicolegal fears.

Stiell et al. recognized this preference when deriving the OKRs: a second rule with a much higher specificity (allowing more substantial reduction in radiography) could have been devised but only at the expense of lower sensitivity. They believe that this decreased sensitivity would be unacceptable to patients and physicians because a small number of fractures would be missed [1]. A later study was performed to validate the OKR, and prospective validation analyzing 1,096 patients found it to be $100 \%$ sensitive for identifying knee fractures [1,2]. The decision rule was interpreted correctly $96 \%$ of the time, and, when applied, the probability of missing a fracture was zero [1]. The decision rule was $100 \%$ sensitive for identifying fractures in patients aged 18 years who were not referred from other hospitals, returned for reassessment, had knee injuries for seven days, or had isolated skin lesions.

This study shows the rules to be an effective tool in deciding which patients with isolated acute knee injury do not require knee radiography when used by doctors in ED with. Also, it shows that ED clinicians do not seem to be adherent to using any specific criteria for ordering plain radiography when acutely reviewing knee injuries. Previous studies have shown a reduction in the number of radiographs requested after the introduction of the OKR [4-8].

In our study, the Ottawa rule was found to have a sensitivity of $100 \%$ and a specificity of $39 \%$. If the OKR was 
followed exclusively, 38 radiographs could have been avoided, with no missed fractures. All of the 12 fractures in this study were identified using radiography, and no further investigation were needed.

It is important for radiologists and emergency medicine physicians to understand that patients can have radiography safely deferred (as used by Stiell et al.). Patients whose symptoms resolve have no need for radiography, but in patients with persistent pain, radiography may be necessary. Patients should be instructed to return for further evaluation, including possible radiography, if symptoms do not improve in five to seven days.

One of the limitations of our study is that we did not follow up with the patients to see if further imaging investigations were obtained or who did not receive radiography at the time of ED presentation, and it is possible that not all patients with fractures were identified. Another limitation is the sample size. Relatively small numbers of patients were included, and accuracy might have been overestimated. Additionally, the definition of any criterion might be differently comprehended by different physicians and advanced nurse practitioners (ANPs).

\section{Conclusions}

In summary, we found the OKRs to have high sensitivity and a reliable screening technique in patients with acute blunt knee trauma. However, compliance with the OKR among academic ED healthcare providers is poor. Improving compliance will require a comprehensive approach involving both education (of physicians and ANPs) and system interventions.

\section{Additional Information}

\section{Disclosures}

Human subjects: Consent was obtained by all participants in this study. Animal subjects: All authors have confirmed that this study did not involve animal subjects or tissue. Conflicts of interest: In compliance with the ICMJE uniform disclosure form, all authors declare the following: Payment/services info: All authors have declared that no financial support was received from any organization for the submitted work. Financial relationships: All authors have declared that they have no financial relationships at present or within the previous three years with any organizations that might have an interest in the submitted work. Other relationships: All authors have declared that there are no other relationships or activities that could appear to have influenced the submitted work.

\section{References}

1. Stiell I, Greenberg G, Wells G, et al.: Prospective validation of a decision rule for the use of radiography in acute knee injuries. JAMA. 1996, 275:611-615. 10.1001/jama.1996.03530320035031

2. Stiell I, Wells G, McDowell I, et al.: Use of radiography in acute knee injuries: need for clinical decision rules . Acad Emerg Med. 1995, 2:966-973. 10.1111/j.1553-2712.1995.tb03123.x

3. Tandeter HB, Shvartzman P, Stevens MA: Acute knee injuries: use of decision rules for selective radiograph ordering. Am Fam Physician. 1999, 60:2599-2608.

4. Jackson JL, O’Malley PG, Kroenke K: Evaluation of acute knee pain in primary care . Ann Intern Med. 2003, 139:575-588. 10.7326/0003-4819-139-7-200310070-00010

5. Bachmann LM, Haberzeth S, Steurer J, ter Riet G: The accuracy of the Ottawa knee rule to rule out knee fractures: a systematic review. Ann Intern Med. 2004, 140:121-124. 10.7326/0003-4819-140-5-20040302000013

6. Konan S, Zang TT, Tamimi N, Haddad FS: Can the Ottawa and Pittsburgh rules reduce requests for radiography in patients referred to acute knee clinics?. Ann R Coll Surg Engl. 2013, 95:188-191. 10.1308/003588413X13511609954699

7. Mitchell PD: The assessment of acute knee injuries by senior house officers in the accident and emergency department. Injury. 1999, 30:215-218. 10.1016/S0020-1383(98)00242-3

8. Stiell I, Wells G, Hoag R, et al.: Implementation of the Ottawa knee rule for the use of radiography in acute knee injuries. JAMA. 1997, 278:2075-2079. 10.1001/jama.1997.03550230051036 